\title{
¿Los de arriba y los de abajo?: Análisis comparativo de las políticas urbanas en los distritos de EI Agustino y San Isidro en Lima, Perú
}

\section{The ones above and the ones below: Comparative analysis of urban policies of the districts of El Agustino and San Isidro in Lima, Peru}

Gelin Espinoza Prado (*)

Pontificia Universidad Católica del Perú

ORCID: 0000-0002-7658-6007

\section{Jimena Limay Castillo (**) \\ Pontificia Universidad Católica del Perú \\ ORCID: 0000-0001-8205-9273}

Fecha de recepción: 31 de enero

Fecha de aceptación: 8 de mayo

ISSN en línea: $2415-2498$

Espinoza Prado, G., \& Limay Castillo, J. (2018) «¿Los de arriba y los de abajo?: Análisis comparativo de las políticas urbanas en los distritos de El Agustino y San Isidro en Lima, Perú». Politai: Revista de Ciencia Política, Año 9, primer semestre, № 16: pp. 13-39.

DOI: https://doi.org/10.18800/politai.201801.001

* Politóloga y asistente de investigación de la Pontificia Universidad Católica del Perú. Miembro de la Sección de Políticas en Seguridad y Anticorrupción del Grupo de Investigación de Políticas Públicas de la Escuela de Gobierno de la misma universidad e investigadora de la Red de Trabajo sobre Sistemas Penitenciarios de las Américas del John Jay Collage of Criminal Justice. espinoza.g@pucp.edu.pe

** Politóloga de la Pontificia Universidad Católica del Perú. Miembro del Grupo de Investigación en Instituciones, Políticas y Ciudadanía de la misma universidad. Internship en la Organización Panamericana de la Salud Organización Mundial de la Salud de Naciones Unidas. imena.limay@pucp.edu.pe 


\section{Sumilla}

El espacio público se ha convertido, recientemente, en el protagonista de las transformaciones urbanas; sobre todo, por ser aquel en el que se han expresado las complejidades de la globalización. Así, los aglomerados urbanos disputan hoy espacios de liderazgo de distintas naturalezas (financieros, culturales, etc.), lo que hace que las ciudades y sus gobiernos se constituyan en terreno fértil para impulsar cambios y, a su vez, son escenarios en los que se expresan todas las contradicciones sociales. El presente artículo analiza las políticas urbanas desplegadas por dos distritos de Lima Metropolitana: El Agustino y San Isidro. Los distritos a estudiar fueron escogidos debido a que su población cuenta con recursos económicos distintos. Mientras San Isidro se caracteriza por ser uno de los distritos limeños con los ingresos económicos más altos, El Agustino es considerado como un distrito con una población con renta media baja. Nuestro modelo de análisis ofrece criterios para poder examinar de manera comparada el fenómeno de la gobernanza urbana en dos distritos significativamente distintos. Con las evidencias, se concluye que pese a las diferencias significativas en el tipo de políticas implementadas, ambos distritos necesitan aplicar mejoras en los espacios degradados para permitir la accesibilidad universal y la redistribución de oportunidades entre la población. Estos retos plantean la necesidad de definir una nueva agenda urbana donde el enfoque feminista y de diseño universal se encuentren presentes para la implementación de políticas concretas que permitan el buen funcionamiento del conjunto del servicio público. Para lograr nuestro objetivo, se empleó una metodología de corte cualitativa, en la que se revisaron documentos de gestión, páginas webs y a la vez, observaciones del espacio urbano, así como un conjunto de entrevistas semiestructuradas a autoridades y ex autoridades municipales.

Palabras clave: Políticas urbanas, gobernanza urbana, políticas públicas. 


\begin{abstract}
The public space has recently become the protagonist of urban transformations, especially because it expresses the complexities of globalization. Therefore, urban agglomerates are currently contesting leadership spaces of different natures (financial, cultural, etc.). This situation turns cities and their governments into fertile ground to promote changes and also into scenarios in which all social contradictions are expressed. The present article analyzes the urban policies deployed by two districts of Metropolitan Lima: El Agustino and San Isidro. The districts to be studied were chosen by virtue of their different income levels: while San Isidro is one of the districts of Lima with the highest income, El Agustino is considered as a low-income district. Our analysis model offers criteria to examine in a comparative way the phenomenon of urban governance in two generally different districts. With the evidence, we conclude that despite the significant differences in the type of implemented policies, both districts need to improve the degraded spaces in order to allow the universal accessibility and the redistribution of opportunities among the population. These challenges pose the need to define a new urban agenda where a feminist and universal design approach is present for the implementation of policies that allow the proper functioning of public services. In order to achieve our objective, a qualitative methodology was applied; it required the review of management documents, web pages and, at the same time, observations of the urban space, as well as a set of semi-structured interviews to authorities and former municipal authorities.
\end{abstract}

Keywords: Urban Policies, Urban Governance, Public Policies. 


\section{Introducción}

«¿Para quién está diseñada Lima?» es una pregunta que, a pesar de la necesidad de mantenerla como un cuestionamiento constante, parece estar muy poco incluida en el debate actual para el diseño de la ciudad. Parte de los objetivos de los gobiernos municipales debería ser garantizar que el derecho a la ciudad sea un derecho humano para todas las personas. Para esto debe de proveer barrios que tengan en cuenta la diversidad real que caracteriza a los espacios urbanos. Pensar el espacio urbano para todas y para todos es hacerlo desde la diferencia, pero no desde la desigualdad: una diferencia entre mujeres y hombres, entre clases, entre orígenes, culturas, religiones, etc. posicionando en igualdad de condiciones todas las demandas, sin decisiones a priori, que excluyan la experiencia de las personas como fuente fundamental de conocimiento en las decisiones urbanas (Muxí y otros 2011).

Es así que el sujeto y sus experiencias aparecen, en la actualidad, no solo como ejes centrales en el planeamiento urbano, sino también como exigencia frente a dos factores relevantes: el orden político y técnico. Por un lado, la administración pública decide según criterios políticos o criterios técnicos las medidas a ejecutarse; sin embargo, los dos criterios en cuestión no están exentos de construcciones sociales, que son el reflejo de formas de vida y manera de entender el mundo que no siempre incluyen visiones, prioridades y la diversidad de toda la sociedad (Sánchez s/f.). Por ello, para contrarrestar el malestar de la ciudadanía es necesario que las autoridades políticas establezcan mecanismos de participación ciudadana para que el diseño urbano respete las lógicas de convivencia de las personas y responda las aspiraciones y deseos de toda la población, sobre todo de aquellas personas que menos voz tienen en los procesos de toma de decisiones y se les pueda garantizar el acceso universal a los servicios de la ciudad.

El presente texto tiene como objetivo analizar las políticas urbanas desplegadas por dos distritos de Lima Metropolitana, El Agustino y San Isidro. Los distritos a estudiar fueron escogidos por contar con una población con recursos económicos distintos. Mientras San Isidro se caracteriza por ser uno de los distritos limeños con los ingresos económicos más altos, El Agustino es considerado como un distrito con una población con renta media baja. El modelo de análisis empleado dispone criterios para poder examinar de manera comparada el fenómeno de la gobernanza urbana en dos distritos significativamente distintos. Con las evidencias, se concluye que pese a las 
diferencias significativas en el tipo de políticas implementadas, ambos distritos requieren de la aplicación de medidas que permitan las mejoras de los espacios degradados para permitir la accesibilidad universal y la redistribución de oportunidades entre la población. Estos retos plantean la necesidad de definir una nueva agenda urbana donde los enfoques feminista y de diseño universal se encuentren presentes para la implementación de políticas concretas que permitan el buen funcionamiento del conjunto del servicio público.

En este sentido, el texto se divide en tres secciones. La primera presenta la metodología empleada en el artículo. En segundo lugar, se presenta la discusión teórica sobre las políticas urbanas. En tercer lugar, se exponen los hallazgos de la investigación de manera comparada, para finalmente presentar las conclusiones y reflexiones.

\section{Metodología}

El presente artículo es de tipo cualitativo y, en este sentido, considera los casos de dos distritos de la capital: San Isidro y El Agustino. Estos han sido elegidos considerando que el resultado del análisis aplicado a cada uno de estos casos enriquece el estudio a través del contraste en la disponibilidad de recursos económicos existente entre ellos ${ }^{1}$.

El artículo utiliza los datos obtenidos como resultado de la aplicación de las siguientes herramientas de recopilación de información:

- Se realizaron entrevistas semiestructuradas a representantes de cada uno de los municipios. Para el caso de San Isidro, la entrevista fue aplicada a un representante de la Gerencia de Planeamiento Urbano; mientras que, en el caso de El Agustino, la entrevista se aplicó a regidores y ex regidores del municipio.

1 Para el presente año, la Ley de Presupuesto del Sector Público destinó 36' 311, 604 soles para el municipio de El Agustino; mientras que, para el caso de San Isidro, este monto ascendió a 221' 529, 807 soles. 
- El artículo plantea a la observación in situ como un recurso de importante valor ya que, a través de lo que se considera como un patrullaje urbano, se ha corroborado la diferencia existente entre la experiencia del uso del espacio público en ambos casos. Para ello, la observación in situ se realizó en avenidas principales, centros de esparcimiento, parques y zonas limitantes con otros distritos, tanto en horas de la mañana como por la noche, para poder observar la variación del número de usuarios en el transcurso del día ${ }^{2}$. El ejercicio de esta observación se enfocó, a su vez, en la identificación de obras municipales que motiven el uso del espacio público de forma segura e inclusiva.

A la par, la información obtenida tras la aplicación de la observación fue comparada con los planes urbanísticos disponibles en las plataformas virtuales de los municipios con el propósito de recolectar evidencia de los enfoques que pudieran haber sido incluidos en la planificación.

\section{Humanizar las ciudades: una mirada desde Jane Jacobs y el enfoque feminista}

Para el presente artículo definiremos habitar como la capacidad para «poder desarrollar las diferentes esferas de la vida en igualdad de oportunidades» (Muxí y otros 2011: 107). Por lo tanto, habitar es mucho más que una suma de actividades como el trabajo, la residencia, el ocio, la educación, etc. La definición nos obliga a replantearnos la idea de ciudad que por mucho tiempo ha sido concebida como un espacio neutro que asigna las mismas oportunidades a toda la población (Col.lectiu Punto 6 2016). El espacio urbano es el «escenario de un conjunto de desigualdades y al mismo tiempo reproduce las desigualdades» (Col.lectiu Punto 6 2016:15). Esta configuración ha priorizado roles, prioridades y jerarquías existentes en la sociedad. Por lo tanto, los espacios urbanos se han construido bajo el supuesto de un modelo estándar de ciudadano, es decir, no se ha tenido en cuenta las experiencias y las necesidades de otros sujetos (Muxí y otros 2011; Col.lectiu Punto 6 2016).

${ }^{2}$ La observación in situ fue realizada entre los meses de agosto a octubre del año 2017. 
El panorama actual no es un fenómeno reciente. Históricamente, el urbanismo ha «tenido multiplicidad de enfoques dependiendo de los momentos sociopolíticos y de la ideología de quien toma las decisiones (funcionalismo, smart cities, urbanismo preventivo, etc.)» (Col.lectiu Punto 6 2016:12). Esto ha generado que por muchos años, la voz de los que ocupan las ciudades sea silenciada, sin embargo, con las nuevas condiciones de desarrollo, el fin de fordismo y la desindustrialización en muchas de los países occidentales a fines del siglo XX, se comienza a cuestionar las formas de intervención del Estado. Esto, a su vez, genera nuevas concepciones no jerarquizadas del urbanismo, las cuales empiezan a considerar los puntos de vista de personas no expertas, es decir, adquieren un componente democrático (Miranda y Gutiérrez s/f). Como ejemplo tenemos el texto de Davidoff, en «Advocacy Planning», donde se plantea que el urbanismo no necesariamente tiene que ser tecnocrático, dándole valor al «componente participativo desde abajo que reconoce el valor del conocimiento no experto» (Sánchez de Madariaga 2008: 76).

En ese contexto, sin tener título universitario ni educación especializada, Jane Jacobs llegó a ser editora de la revista Architectural Forum. Pese a ser ridiculizada por los tecnócratas del urbanismo moderno, Jacobs, desde su pequeño espacio, criticó duramente las tendencias del urbanismo autoritario y deshumanizado impuesto de arriba hacia abajo (Plataforma Urbana 2016). Podemos resumir su legado en cinco ejes: a) el cuidado en el diseño del espacio público, b) la protección del patrimonio arquitectónico y urbano, c) los peatones como prioridad, d) la densidad equilibrada y, e) la multiplicidad de usos que tienen los espacios.

La propuesta disruptiva de Jacobs (2011) sostenía que antes de realizar algún cambio o modificación en la ciudad era necesario conocer el espacio a profundidad. Ello implica conocer cómo se usan los espacios, las actividades que se realizan en ellos, es decir, conocer la trama urbana para que los cambios que se planea introducir no rompan con las costumbres del tejido social. Uno de los puntos más importantes de esta sección es la fuerte crítica que se le hace al urbanismo de los tecnócratas que tomaban las decisiones sobre los cambios en la ciudad no solo sin participación ciudadana, sino de manera autoritaria. Jacobs introduce la idea de participación urbana como eje 
central. No existe un modelo único de ordenamiento urbano; este depende de las formas de convivencia y de relación de las personas con su entorno.

En segundo lugar, la autora fue una gran protectora del patrimonio arquitectónico y urbano. Esto se evidencia en su lucha contra la prepotente política de autopistas que ingresaban en la ciudad y lo arrasaban todo. Esta defensa del patrimonio frente al desmedido incremento de las pistas se aprecia en su defensa por salvar el Village, su barrio en New York y finalmente en Toronto, Canadá.

En tercer lugar, y vinculado al segundo punto, tenemos su defensa del peatón. Para la autora, es esencial recuperar la vitalidad de las calles, pues ellas no son un mero vacío para la movilidad. La calle es para Jacobs una auténtica y compleja institución social donde desde niños aprendemos a socializar y construir comunidad. Si la calle termina privilegiando al automóvil por sobre el peatón, entonces la calle muere y allí comienza el fin de la ciudad.

En cuarto lugar, la densidad equilibrada es para Jacobs un factor importante cuando se habla de seguridad. La autora propone tener una delimitación clara entre los espacios públicos y privados; manzanas no muy grandes que generen diversos espacios de encuentros (por ejemplos las esquinas y los cruces en las calles), donde se generaría un ambiente de seguridad natural a generada por las personas que habitan los espacios públicos. Jacobs defiende la densidad y la vida en comunidad. Sostiene que allí está la cura de la inseguridad y la violencia; conocer al vecino, conformar redes, mezclarnos con los diferentes, saludarnos y volver a reír en el espacio público (Plataforma Urbana 2016).

Finalmente, Jacobs se refiere a la multiplicidad de usos que tienen los espacios públicos. Las calles sirven para hablar y conocerse. Existe ahí un entramado de vínculos, relaciones y contactos que una ciudad genera entre sus habitantes. Todas las premisas expuestas han hecho de Jane Jacobs una de las mujeres más influyentes en el urbanismo actual. Jacobs nos permite concebir una ciudad más humanizada, priorizando la trama urbana sobre el ordenamiento.

Ahora bien, un enfoque disruptivo más reciente es el presentado por las feministas urbanas. El urbanismo feminista sostiene que las ciudades y 
pueblos se han construido sin tener en cuenta la sostenibilidad en la vida y los vínculos con el entorno. Frente a ello, este nuevo enfoque coloca en el centro de las decisiones urbanas a la vida cotidiana ${ }^{3}$ de las personas. Resalta además que dentro de las ciudades existe una diversidad de las experiencias y sujetos, se opone a la idea universal de sujeto. Este punto es un salto significativo cuando se trata de pensar en una ciudad para todos en las que personas con capacidades especiales pueden vincularse de manera más amigable con los espacios públicos. De manera adicional, el enfoque feminista analiza cómo los roles de género influyen en el uso y disfrute de las ciudades.

Es así que el enfoque feminista resalta cinco ideas importantes. Una primera tiene que ver con la proximidad de los espacios, satisfacer las necesidades cotidianas en el menor tiempo posible y con recorridos cortos. Una segunda idea está vinculada a la diversidad de las personas tanto en el género, raza, diferencias físicas, entre otros; esta idea reconoce que las personas no son sujetos uniformes, sino que tienen capacidades diferentes de relacionarse con el entorno. En tercer lugar, se resalta la idea de autonomía que deben de tener las personas. Se reconoce que no existe un modelo universal de sujeto. Se acepta que dentro de las ciudades viven personas invidentes, en sillas de ruedas, con problemas auditivos, etc. El objetivo del enfoque feminista es que todas las personas, pese a sus limitaciones puedan desarrollarse e interactuar con el mundo sin barreras que puedan impedir su participación plena y efectiva. La autonomía también se extiende al plano de la seguridad, en la que se plantea la creación de espacios que puedan garantizar, sobre todo a las mujeres, sentirse seguras. El cuarto punto es la vitalidad de las calles (entendida también como vida en la calle) que está vinculada a la idea de seguridad. Rescatamos aquí la idea de Jacobs de «los ojos de la calle», una vigilancia natural que se genera en el espacio urbano cuando las personas se apropian de los espacios públicos. Finalmente, el enfoque resalta la importancia de la participación ciudadana en la planificación urbana.

La planificación urbana es un trabajo transversal, transescalar, interdisciplinario y con constante coordinación con la ciudadanía. En ella, las decisiones no se toman únicamente en base a información estadística que

\footnotetext{
${ }^{3}$ El concepto de vida cotidiana engloba el desarrollo de todas aquellas actividades que desarrollamos en nuestro día a día o eventualmente. La vida cotidiana no se puede desvincular de un espacio compartido y de un tiempo finito.
} 
distorsiona la distribución territorial e invisibiliza las necesidades que cada población tiene.

En esta sección se han presentado, a modo de resumen, un debate teórico bastante amplio que gira entorno a la diversidad de sujetos, la importancia de la vida cotidiana y la importancia de la participación ciudadana en la planificación urbana. En las siguientes secciones, analizaremos un caso comparado para apreciar la pertinencia del presente debate teórico en dos distritos de Lima Metropolitana.

\section{El Agustino: distrito provinciano}

El Agustino encontró su nombre mucho antes de su establecimiento oficial, cuando en 1551 los religiosos de la Orden de San Agustín llegaron al Perú. En sus inicios, la zona albergó las haciendas de las familias criollas e indígenas. Allí se trabajaba la tierra para producir maíz y alfalfa y, se confeccionaban ladrillos y adobes. Con el paso de los años, en 1947, en el distrito se fundan las primeras barriadas producto de las migraciones internas.

Hoy, El Agustino cuenta con una población de 193319 habitantes (INEI 2015), lo que representa solo el 2,14\% de la población total de Lima Metropolitana. El Agustino, reúne diversas caras de la Ciudad de los Reyes, por un lado el viejo rostro del distrito con todos sus problemas sociales y por otro, el acercamiento del avance económico con la instalación de centros comerciales y negocios.

Al igual que Lima Metropolitana, El Agustino ha tenido «dificultades para convertirse en un espacio de referencia e identidad para sus ciudadanos, ya sea por la ausencia de proyectos políticos compartidos o por la dificultad para integrar a los distintos sectores y capas sociales que han ido poblando» (Bensa 2015:215). Desde el regreso a la democracia y, de manera más precisa, desde las elecciones regionales y municipales 2002, las intervenciones municipales se han centrado en proyectos y obras de infraestructura, dejando de lado una visión de conjunto e integral de El Agustino como distrito. Esto ha generado que por mucho tiempo el distrito esté sin pistas, lo cual obstaculizaba su integración y la interconectividad con 
la ciudad. Por otro lado, El Agustino ha ganado una notable fama por ser uno de los distritos con mayor incidencia delincuencial por la presencia de pandillas y barras bravas (Plan de Gobierno 2002).

Al revisar el plan de trabajo de los últimos alcaldes del distrito, se contemplaba siete ejes de acción priorizada por los líderes políticos (Gráfico 1). Para objetivos del estudio, nosotros nos centraremos en los siguientes tres ejes: desarrollo urbano y vivienda, infraestructura básica y equipamiento social.

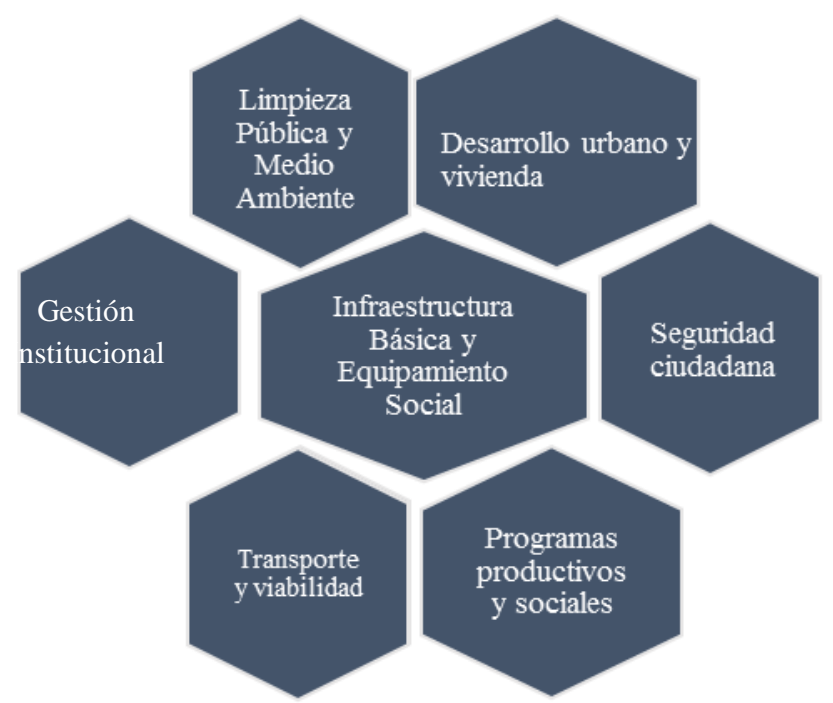

Gráfico 1. Ejes del plan de trabajo 2002 -2019. Fuente: Elaboración propia en base al Plan de Gobierno Municipal 2002, Plan de Gobierno Municipal 2007 y Plan de Gobierno Municipal 2012.

Ahora bien, al analizar la situación del Agustino respecto a infraestructura básica y equipamiento social llegamos a las siguientes aproximaciones. En primer lugar, desde el 2006 se inició un acondicionamiento de los centros comerciales, se construyeron más espacios de recreación e integración de la ciudadanía. Por otro lado, se impulsó la articulación de las zonas urbanas aisladas de los cerros, mediante la habilitación de circuitos viales y obras de infraestructura. Los alcaldes municipales, durante su gestión buscaron integrar las distintas zonas del distrito y, a la vez, organizarlo. Entonces, 
vemos que aquí se refuerza la idea del centro como eje principal para el funcionamiento del distrito. Se entiende que es necesario integrar a las zonas aisladas para concretar el desarrollo y promover también los flujos comerciales, interpelando la idea utópica de que la vida en el núcleo urbano puede ser donde coexistan todos los actores de una ciudad.

Pero, al realizar la observación del espacio urbano, se pudo apreciar que una tendencia creciente en el distrito es la aparición de rejas y agentes particulares de seguridad ${ }^{4}$. Esta medida es una respuesta a la creciente percepción de criminalidad, ya que al revisar los datos estadísticos se observa que en los últimos diez años, el índice de inseguridad ha disminuido ${ }^{5}$ (INEI 2017). En términos teóricos, esta respuesta puede ser comparada a lo expuesto por Evan Mackenzie (1996) que estudia el desarrollo de las privatopías como una patología urbana producto de la fragmentación y la falta de capacidad de gestión de las instituciones estatales.

Las privatopías se oponen a la idea de crear una seguridad natural en las calles. El aumento de las rejas disminuye la actividad de los vecindarios, por lo tanto, hay menos oportunidades para mirar lo que pasa en el entorno. Recordemos que para Jacobs «las calles son los principales órganos de una ciudad y que una vereda urbana en sí misma no es nada, que es una abstracción, la que adquiere significado en conjunción con los edificios y usos que se ubican en el borde» (Miranda y Gutiérrez s/f: 8), en otras palabras, para Jacobs el contacto social es la base de una convivencia civilizada en comunidad.

En segundo lugar, respecto al desarrollo urbano y vivienda, encontramos que en las últimas gestiones se flexibilizó las normas de zonificación y edificación para que las personas puedan construir sus viviendas en los cerros, pero a la vez, no se descuidó criterios de seguridad. Así también, formalizó las áreas urbanas irregulares, rehabilitando las viviendas precarias y erradicando los tugurios existentes en el distrito. Para que los vecinos tengan asesoría legal y un control del crecimiento de las viviendas en los cerros, creó una oficina de monitoreo. Finalmente, la gestión promovió el

\footnotetext{
${ }^{4}$ La investigadora vive en el distrito por lo que se firma que el incremento de rejas en el distrito se inició aproximadamente en el 2010.

${ }^{5} \mathrm{Si}$ bien es cierto que los índices han disminuido visiblemente, el distrito aún se ubica entre los lugares con mayores niveles de violencia y muertes violentas del país
} 
acceso de créditos en la construcción de viviendas. Algo que se puede ver en el distrito es la presencia de la autoconstrucción que se inicia desde la llegada de las primeras barriadas al lugar pero que hasta ahora persisten ${ }^{6}$. Las evidencias (y las conversaciones con las familias más antiguas del distrito) nos lleva a decir que el alcalde en su periodo de gestión buscó la mejorar en la calidad de las viviendas de las personas. El gobierno municipal flexibilizó algunas medidas. Con ello, buscaba responder a una demanda urgente de la población que impactaba directamente en su calidad de vida. Pero no ordenó el distrito, ya que la medida careció de planes y procesos de habilitaciones urbanas sostenibles para la conformación de conjuntos habitacionales e industriales adecuados a una normatividad técnica.

Había una zona por La Menacho II en la que las personas vivían en casas prefabricadas, las que por el tiempo se encontraban en pésimo estado. La falta de limpieza y los bajos niveles económicos de las familias que vivían ahí había generado que en las noches los fumones vayan a drogarse al lugar, luego robaban. No podíamos tenerlo en ese estado. Luego de casi tres años de conversaciones, los convencimos de abandonar el lugar. Les dimos unos bonos de vivienda, como iniciales para que puedan adquirir los departamentos que se estaban construyendo en Los Parques. Ahora todos ellos viven mejor. Era una medida que se tenía que hacer. Quedaron felices todos, ellos con casas de calidad y nosotros también porque destruimos el fumadero y metimos a Serenazgo para capturar a esos palomillas que robaban. (Entrevista a ex regidor 2)

Ahora bien, al indagar los criterios que se usaron para la ejecución de las medidas y el nivel de participación de la ciudadanía en la toma de decisiones pudimos constatar que estas fueron implementadas por criterios netamente técnicos.

Nos hubiese gustado tener más participación de las personas, pero salimos de un periodo duro [hace referencia al regreso a la democracia] y las personas uno desconfían mucho y dos, no teníamos tiempo de esperar que ellos decidan asistir a las reuniones. Además, en ese tiempo el tema de participación no era tan fuerte como ahora. Nosotros necesitábamos actuar ya. (Entrevista a ex regidor 2)

${ }^{6}$ Según informes municipales, el $65 \%$ de las viviendas del distrito son de material noble. 
Recordemos que la participación ciudadana está relacionada a mecanismos de democratización del poder local y mayor poder decisorio de la sociedad (Bensa 2015:289). Consultar con la población es importante también porque permite ejecutar las medidas de ordenamiento urbano respetando la forma en la que el ciudadano se relaciona con su entorno, en palabras de Jane Jacobs, construir y ordenar [o desordenar] la ciudad respetando el trama urbano. Es así que la planificación urbana de El Agustino fue ejecutada de arriba-abajo top down. Se trató de proyectos urbanos ejecutados de manera autoritaria y sin participación de la ciudadanía que es el eje central de la vida urbana (Jacobs 2011). La gestión buscó la funcionalidad. La planificación estratégica se centró en resolver los problemas concretos y el diseño espacial, dejando de lado el uso que las personas le darían. No se contempló a las personas como sujetos no uniformes y con capacidades diferentes para relacionarse con el entorno.

En la misma línea, desde el enfoque urbanista, la ausencia de participación - bottom up- no permitió contemplar la diversidad de identidades y experiencias para construir y ordenar el distrito. Si bien es cierto, se buscó generar espacios más integrados y relacionados, como lo propone el urbanismo feminista, las gestiones de los últimos alcaldes ${ }^{7}$ no han cumplido a cabalidad los cinco puntos que plantea el urbanismo feminista, los cuales son: la proximidad de los servicios, la comprensión de la diversidad, la autonomía de la ciudadanía (entendida como accesibilidad universal y seguridad), vitalidad y vida en las calles y la representatividad. De estos cinco ítems, lo más ausentes fueron la accesibilidad universal y la vida en las calles. Dentro del plan de los últimos alcaldes no se incluyen a las personas con capacidades distintas y eso se evidencia en la ausencia y mal estado de las rampas, el tamaño reducido de las veredas, etc. ${ }^{8}$

Un cambio importante para el distrito se inicia en el 2007, cuando el ex cuartel Militar La Pólvora es demolido para dar vida al primer megaproyecto de vivienda del distrito, «Los Parques de El Agustino». La medida fue implementada por el presidente Alan García, quien planeaba construir 2600 viviendas sobre los 227 mil metros cuadrados del ex cuartel. Los

\footnotetext{
${ }^{7}$ Alcalde Víctor Salcedo Ríos (2002 - 2014) y el Alcalde Richard Soria Fuerte (2014 - 2018).

${ }^{8}$ El problema de accesibilidad para las personas con capacidades especiales se mantiene hasta la actualidad, en la que el alcalde no ha ejecutado medidas para crear espacios más amigables para las personas con capacidades y necesidades diferentes.
} 
departamentos fueron vendidos por el Crédito Mivivienda ${ }^{9}$ y Techo Propio ${ }^{10}$ (Actualidad Empresarial 2007). Inicialmente, el proyecto consistía únicamente en la construcción de viviendas. Sin embargo, una parte del terreno fue entregado para la construcción del primer centro comercial del distrito, «Agustino Plaza». Dicho centro comercial fue inaugurado el 29 de octubre de 2011 y generó que no solo más de 90 locales comerciales se instalaran en el distrito, sino también que se mejore la seguridad y el cuidado del espacio público.

El megaproyecto se construyó sobre un área en la que la precariedad estaba muy presente, tal como lo explica uno de nuestros entrevistados:

Los Parques [haciendo referencia a los condominios y al centro comercial] se han levantado sobre un terreno donde la pobreza reinaba. A la zona se le conoce como Ancieta. Antes, la mayoría de las casas eran de adobe y algunas ni tenían agua ni desagüe. Además, como está cerca de Barrios Altos, habían bastantes robos y delincuencia. El alumbrado público era pésimo. Habían zonas oscuras y no habían pistas, solo era tierra. Pero desde que llegaron Los Parques todo cambió, el precio de los terrenos se fueron a las nubes [subieron de precio], pavimentaron todas las pistas, pusieron más iluminación, las casas comenzaron a crecer, la gente puso sus negocios, más seguridad, más limpieza. Esta zona se volvió el centro de El Agustino. Nadie imaginaba que una de las zonas más marginadas se volvería en lo que hoy usted ve, una zona en la que las familias salen a pasear. (Entrevista a ex regidor 2)

\footnotetext{
${ }^{9}$ Es un crédito inscrito al Ministerio de Vivienda, Construcción y Saneamiento que está supervisado por la Superintendencia de Banca, Seguros y AFP (SBS) cuyo objetivo principal financia la adquisición de una vivienda nueva o usada o la construcción de una vivienda con terreno propio; va dirigido a personas que no cuenten con un inmueble, tampoco pueden ser dueños los hijos menores de edad, ni el cónyuge. Se financian viviendas que cuesten desde s/. 53,900 a s/. 269,500 con una cuota inicial mínima del 10\% sobre el precio del inmueble. Cabe resaltar que; no todas las entidades que financian la compra de una vivienda financian la construcción, las viviendas adquiridas pueden estar "en planos" sujetas a un periodo de gracia donde si existe el cobro de intereses y que el plazo de pago es de 10 a 20 años con cuotas fijas. Este crédito cuenta con el beneficio del BBP (Fondo Mivivienda 2015).

10 Es un programa del Fondo Mivivienda, del Ministerio de Vivienda, Construcción y Saneamiento. Techo Propio financia la adquisición, construcción o mejoramiento de una vivienda; va dirigido a familias con ingresos menores a s/. 1,860. Este crédito cuenta con un subsidio directo que no se devuelve llamado Bono Familiar Habitacional (BFH) que varía de acuerdo a la modalidad fluctuando entre s/. 8,855 y s/. 19,250. El proceso para acceder a este préstamo requiere la inscripción del grupo familiar en las oficinas del Fondo Mivivienda previa evaluación del Sistema de Focalización de Hogares (SISFOH), siendo indispensable el no haber recibido ayuda habitacional previa del Estado y contar con un ahorro mínimo. Las entidades que brindan este crédito son algunos bancos, Cajas Municipales de Ahorro y Crédito (CMAC) y financieras. El valor máximo de la vivienda es el equivalente a 14 UIT (s/. 53,200) (Fondo Mivivienda 2015).
} 
Este testimonio, contrastado con conversaciones informales sostenidas con los vecinos del distrito, nos permite dar cuenta de dos detalles importantes. El primero tiene que ver con las externalidades positivas generadas por la inversión privada: acceso a servicios básicos como el agua y desagüe, mejora en la infraestructura del lugar (mayor iluminación, mejora en pavimento, etc.) y reducción del miedo al crimen (esto vinculado a la presencia de seguridad privada y cámaras de seguridad). Por otro lado, las mejoras en las condiciones del lugar y la oferta comercial han generado que las personas tengan más presencia en el espacio público. Pero este impulso por salir a las calles por parte de los vecinos agustinianos no ha sido motivada por medidas ejecutas por las autoridades municipales, sino por la inversión privada.

En la introducción sosteníamos que el derecho a la ciudad debe ser un derecho humano en el que todos y todas puedan gozar con igualdad de oportunidades del espacio público. Sin embargo, cuando la autoridad estatal no brinda las facilidades para que el ciudadano pueda gozar plenamente del espacio público y esta medida es motivada por el privado, se cae en dinámicas consumistas en las que solo algunos podrán disfrutar de los servicios que provee el actor privado. Esto hace que espacios públicos sean objeto de uso para disfrute de minorías. Se trata de un espacio público para la élite que cada vez pierde más conexión con la sociedad en su conjunto, más aún si nos referimos a sociedades con diferencias socioeconómicas $\mathrm{y}$ culturales marcadas (López 2014). En la siguiente sección se analiza el caso del distrito de San Isidro, en la que se podrá observar que pese a las características diferentes de los distritos, la planificación que garantice la equidad y el acceso universal sigue siendo un reto para los líderes territoriales de Lima Metropolitana. 


\section{San Isidro, el distrito «soñado»}

Recientemente, un medio de comunicación televisivo de señal abierta emitió un segmento periodístico en el que presentaban al distrito en cuestión y listaban durante 1'30' aproximadamente la calidad de servicios de la que gozaban sus vecinos; «San Isidro, distrito amigable» lo llamaron. Todo limeño sabe que, de principio, es un logro casi impensable que se realice un reportaje sobre un distrito que resalte alguna característica positiva y no se dedique a analizar cómo actúa el crimen organizado en la zona, cuánto ha aumentado el nivel de robos a mano armada o cómo el presupuesto para obras públicas parece desaparecer mágicamente. Tras analizar el caso de El Agustino, San Isidro parece ser, lo que algunos considerarían, «la tierra prometida», ese caso tan lejano y utópico en el que los vecinos pagan sus impuestos y estos se destinan a obras públicas y programas municipales que todos disfrutan; todos felices; fin de la historia. No obstante, ¿cuán cierto es el caso de San Isidro como caso atípico?, ¿realmente existe esta gestión eficiente que propone un nuevo enfoque de participación ciudadana a través del fomento del uso del espacio público? En un intento por responder estas preguntas, consideramos, nuevamente, que la calle, incluso más que el análisis de los planes urbanísticos, nos daría respuestas más certeras.

Sobre el distrito es posible tener en cuenta que posee una población estimada de 56800 habitantes y una extensión de $11,1 \mathrm{~km}^{2}$. Limita con los distritos de Lince, La Victoria y Jesús María hacia el Norte; con el distrito de San Borja por el Este; y con Surquillo y Miraflores hacia el Sur; también limita con el Océano Pacífico hacia el Oeste. Dentro de los lugares más reconocidos del distrito, se encuentran el centro financiero comúnmente llamado Corpac en el que se ubican avenidas como República de Panamá, Rivera Navarrete, Canaval y Moreyra, y Javier Prado. De igual forma, es reconocido el parque El Olivar como punto de reunión y de esparcimiento; característica que comparte con el parque La Pera o el malecón.

El distrito se caracteriza, a su vez, por contar con la convivencia de distintos tipos de arquitectura que le dan una particularidad única. Espacios como el centro financiero poseen edificios de arquitectura contemporánea; mientras que también existen casonas dispersas a lo largo del sector. Dentro de las peculiaridades existentes en sus edificios y construcciones, resalta las grandes casas con tejados a dos aguas como resultado de las migraciones europeas en 
la primera mitad del siglo pasado o casas hacienda que datan del siglo XVII. Cabe mencionar que el distrito alberga un gran número de embajadas y residencias de embajadores dispersas en distintas avenidas.

Dentro de este distrito, transcurren grandes e importantes avenidas como lo son la avenida Javier Prado que une al distrito desde su límite con Magdalena del Mar hasta su límite con San Borja. La avenida Salaverry conecta al Centro de Lima con San Isidro atravesando el distrito de Jesús María hasta llegar al malecón sanisidrino. De igual forma, la avenida Vía Expresa conecta el Centro de Lima con el centro financiero del distrito de San Isidro y, apoyándose en el servicio de transporte ofrecido por el Metropolitano, conecta al distrito con sus pares pertenecientes al Cono Norte como los son los distritos de Independencia y Los Olivos. Por su parte, la avenida Arequipa, en su camino desde Lima Cercado hasta Miraflores, une a San Isidro con estos municipios y con el distrito de Lince.

Por otra parte, San Isidro ostenta un IDH de 0.99, lo que lo ubica en la categoría Muy Alto. Esto, en parte, se puede vincular a los servicios que la municipalidad ofrece que se enfocan en proporcionar respaldo a los sanisidrinos en distintos ejes (Cuadro 1), procurando la calidad de vida de los beneficiarios. Con ello, se genera un enfoque de distrito sostenible en el que considera que los desafíos existentes como gobierno local se deben a que la ciudad de Lima mantiene lineamientos de ciudades de la década de 1950 que priorizan un transporte que permita movilizarse más rápido y, por ende, que se han formado a favor de los automóviles y no de los ciudadanos. En consecuencia con esta postura y con la finalidad de impulsar un cambio localizado de la realidad del desarrollo urbano de la ciudad de Lima, San Isidro impulsa, entre otros, programas tales como +Ciudad, San Isidro Recicla, Cultura Libre, el Plan de Movilidad Urbana Sostenible, junto al proyecto San Isidro: Smart City cuyo propósito principal es la recuperación de los espacios públicos para el uso eficiente de estos según las demandas de los ciudadanos. 


\section{Servicios}

- Salud Humana

- Laboratorio Municipal

- Vigilancia Sanitaria

- Cuna Jardín Municipal

- Mi Bus

- Sistema de Focalización de Hogares
Centros de encuentro vecinal

- Salas de usos múltiples

- Asesoría Legal

- Consultoría nutricional

- Enfermería

- Terapia física y de rehabilitación

- Psicología

- Podología

- Terapias Orientales

\section{Otros}

- Tenencia responsable de mascotas

- Vaso de leche

- OMAPED

- Demuna

- Deporte

Cuadro 1. Servicios proporcionados por la Gerencia de Desarrollo Humano de la Municipalidad de San Isidro. Fuente: Elaboración propia en base al esquema de servicios de desarrollo humano del Plan de Gobierno (2015-2018)

Con base en la teoría del lugar central, es posible, además, considerar a San Isidro como una centralidad. Según el supuesto de localización homogénea de consumidores potenciales, San Isidro podría considerarse como un centro homogéneo por poseer una población que oscila entre los sectores A y B, lo que genera la necesidad de focalizar ciertas ofertas de bienes y servicios en lugares específicos. Aplicándolo en un caso concreto, San Isidro aterriza tal teoría en el caso de su centro financiero. La estrategia de la localización de las empresas en este sector genera una concordancia aproximativa de áreas de mercado que conducen a la yuxtaposición espacial de áreas de venta. El hecho de que se produzcan viajes desde distritos pertenecientes a los conos implica la aceptación de viajes largos para acceder a servicios. La economía, entonces, impulsó la conjunción del uso funcional del espacio y la utilización oportunista del tiempo (Sennett 1994:202). 


\section{¿La «Ciudad sostenible» sostiene a todos?}

El representante de la Gerencia de Planeamiento Urbano de la Municipalidad de San Isidro, considera que el trabajo que está realizando la actual gestión del distrito está dirigido a lograr la diversificación de opciones de uso de los espacios para los vecinos y no limitarlos a un uso contemplativo. A partir de ello, se identifica una postura de reconocimiento del ciudadano como beneficiario primordial $\mathrm{y}$, por tanto, la necesidad de crear un distrito hecho para el usuario del servicio (Jacobs 2011).

La instalación de gimnasios y de juegos infantiles en distintas avenidas y parques parece ejemplificar claramente el ir más allá del discurso y pasar a la ejecución de estas acciones enfocadas en el uso eficiente del espacio público. Podría considerarse, incluso, que el municipio ha adquirido un norte destinado a la inclusión. Ello al poder observar que se ha dedicado esfuerzos al acondicionamiento de las veredas para permitir el libre tránsito de personas en sillas de ruedas en gran número de sus calles y avenidas.

Todo ello resulta incluso más increíble si se tiene en cuenta que la participación ciudadana parece ser una prioridad para la gestión actual. Reuniones con dirigentes de juntas vecinales, participación en la programación del uso del presupuesto, consultas a juntas vecinales para el desarrollo de proyectos, etc. son parte de las modalidades en las que la participación ciudadana tiene cabida en la toma de decisiones del municipio. Sumado a ello, el concurso público para el diseño de obras públicas y la medición de la satisfacción del usuario a través de encuestas aplicadas de forma constante configuran una especie de sistema de retroalimentación que parece funcionar efectivamente y convierte al distrito en uno de los mejores distritos para vivir (Diario Gestión 2017). San isidro, por lo tanto, resultaría ser ejemplo exitoso de la superación del autoritarismo de los tecnócratas contra el que Jacobs tanto luchó y un exponente de que la consulta y el reconocimiento del espacio brindan mejores resultados que la intervención con excesiva especialización.

No obstante el aparente hallazgo de la «fórmula del éxito», encontramos que en San Isidro se produce un interesante fenómeno. Conforme el ejercicio de la observación se aleja del centro, todo lo armonioso y encandilante de San Isidro parece irse agotando y desvanecerse. Los problemas limítrofes que han 
caracterizado las disputas con otros distritos, de forma especial, con Magdalena del Mar podrían explicar esta característica de las periferias como consecuencia de una mala o inexistente distribución acordada del cuidado y mantenimiento de los espacios públicos. Si bien en algunos límites San Isidro parece producir un efecto de contagio del correcto proceso de ejecución del presupuesto público y eleva la calidad del mantenimiento de los espacios públicos de los distritos colindantes, otras áreas limítrofes tienen el mismo efecto, pero, en esta ocasión, el contagio es desventajoso.

Las zonas residenciales, aquellas en las que incluso se encuentra prohibido el tránsito del transporte público, gozan de señalización eficiente para el peatón, seguridad permanente proporcionada por efectivos del Serenazgo y rampas con pendientes funcionales para personas en sillas de ruedas; no obstante, a medida que se transita hacia una avenida principal, la importancia que parecía haber tomado el ciudadano se debilita y todo lo mencionado previamente parece haberse trabajado a la premura, aparentemente con la única intención de cubrir una necesidad nominalmente, pero no en esencia, no priorizando al ciudadano, retornando nuevamente al «vehículo centrismo». Caminar por avenidas como Javier Prado o Arequipa expone claramente esto, ya que resulta sencillo encontrar rampas empinadas, con el pavimento desgastado que genera baches que imposibilitan que una persona en silla de ruedas pueda maniobrar libremente; cruces de cebra mal definidos; semáforos que favorecen el tránsito del transporte vehicular y retiene al ciclista y al peatón; etc.

No se trata entonces, necesariamente, de un problema de mala distribución de labores en las periferias. Esta situación permite abrir un debate sobre el «hermetismo» que suele caracterizar a la gestión distrital en general. Es necesario aclarar a qué se hace referencia al utilizar dicho término con la finalidad de acceder a la conclusión alcanzada con respecto a este caso. El hermetismo que se menciona considera que toda obra pública impulsada por una gestión municipal se realiza para la satisfacción de un sector, pero no del ciudadano. El grupo beneficiario que se reconoce para el diseño y ejecución de una obra pública está delimitado y excluye a quien no se considera parte del conjunto, al que es de afuera, el que es distinto. Cabe preguntarse, por tanto, ¿para quién está pensado San Isidro? 
Más allá de los casos de discriminación que se han suscitado en el distrito, la discusión propone el cuestionamiento de cómo se aborda la administración del espacio público y hasta dónde la delimitación territorial contribuye a la sectorización social. Resulta interesante encontrar en este caso una peculiaridad tal que permite identificar un factor que muy probablemente se repita en el resto de distritos considerados como gestiones eficientes. Sin embargo, ello será tema de análisis de futuros estudios que deseen abordar tal discusión.

Por otra parte, es importante enfatizar que, si bien San Isidro se encuentra en un proceso de inclusión y de fomento del uso del espacio público por los ciudadanos, no existe una medida concreta para asegurar que un porcentaje importante de los usuarios se sienta totalmente satisfecho: la población femenina. Esto resulta crucial ante un contexto en el que la ciudad de Lima ha sido considerada como la quinta ciudad más peligrosa para las mujeres en el mundo (El Comercio 2017). Actualmente, doce distritos de la capital cuentan con ordenanzas que sancionan el acoso callejero; San Isidro no es uno de estos. Tras conversar con el representante de la Gestión de Planeamiento Urbano, este consideró que en el distrito existe una cultura de reportar y denunciar la violencia de género y aseguró que no se ha registrado ninguna denuncia por acoso callejero. Esto, sin embargo, no supone que el acoso y la violencia no existan, sino que, muy probablemente, se encuentran invisibilizados. De todas formas, en una ciudad tan violenta como lo es Lima, se requieren de medidas concretas que no esperen al surgimiento de denuncias.

Se dista así de esa «igualdad de oportunidades» con la que Muxí Martínez et al. (2011: 107) definía el habitar, ya que la población femenina parte de una situación de desventaja como resultado de la estructura social. Reconocemos nuevamente que la idea de ciudad tal como se entiende hoy en día parte de una premisa errada que considera que los ciudadanos parten a ejercer su ciudadanía desde una posición de igualdad, eximiendo la existencia de inequidades inherentes en la sociedad (Col.lectiu Punto 6, 2016). A su vez, el caso sanisidrino dista de aplicar un enfoque feminista pues desconoce la heterogeneidad del individuo y asume una uniformidad del usuario. Nuevamente y al igual que en el caso de El Agustino, San Isidro flaquea en el cumplimiento de la totalidad de los cinco puntos que propone la teoría 
feminista, de manera especial en aquel que plantea la comprensión de la diversidad.

\section{Conclusiones}

Empleando el enfoque de inclusión urbana que considera que las personas no son sujetos uniformes y poseen capacidades diferentes para relacionarse a su entorno, y el enfoque urbanista feminista que señala que la ciudad está compuesta por diversidades, es posible concluir que es necesario diseñar las ciudades no con un único pensamiento espacial, sino considerando el uso que las personas dan a estos espacios.

1. Las tendencias en el proceso urbanístico se diferencian debido al tipo de formación de los distritos. En El Agustino prima la autoconstrucción y la ilegalidad que han producido un crecimiento desorganizado como producto de las migraciones y son escasas las construcciones de arquitectura colonial o previas al siglo XX. San Isidro, por el contrario, posee zonas con grandes construcciones de arquitectura de mediados del siglo XIX e inicios del siglo XXI. De igual forma, posee zonas con arquitectura muy moderna enfocada a la vivienda multifamiliar con grandes complejos departamentales; mientras que El Agustino muy recientemente ha podido experimentar el boom de las construcciones de complejos multifamiliares y villas de viviendas.

2. Existen similitudes en ambos distritos. Si bien es cierto que San Isidro posee un mejor sistema de señalización y estado de rampas y vías de movilización, existen zonas, sobre todo, en los límites con otros distritos, con deficiencias en estos servicios. De igual forma, El Agustino presenta un mal estado de pistas y ubicación precaria de semáforos. Ambos enfrentan muchos retos como distritos porque aún mantienen el enfoque de diseño espacial pensado para priorizar a personas varones, con un tipo específico de capacidades. No obstante, existe en 
San Isidro una apertura más trabajada que en El Agustino para la inclusión de nuevos enfoques.

3. El uso del espacio público para la recreación, la convivencia y el ocio genera en el caso de San Isidro la existencia de un tramo urbano mucho más fuerte que en El Agustino, teniendo este último una población flotante caracterizada por tener una movilidad con motivo laboral. El uso de espacios como parques y centros de interacción es reducido en El Agustino como resultado de la percepción de inseguridad, lo cual, a su vez, genera un círculo vicioso de desconfianza e impide que se den interacciones con los demás.

4. La inversión del presupuesto público en el caso de El Agustino, en su mayoría, está destinada a la construcción de vías y parques. En este sentido, es consecuente con la tendencia a complacer visualmente a través del concreto. Estas obras, sin embargo, no cuentan con el respaldo de estudios premeditados que tomen en cuenta los usos que los ciudadanos le dan al espacio público y las demandas de los mismos. Por su parte, San Isidro invierte en distintas áreas que fomentan la participación ciudadana y la vida digna; no obstante, en lo que respecta al espacio público, se carece de medidas que reconozcan a la ciudadanía en general como beneficiarios y no solo a sectores específicos.

5. El artículo abre dos interrogantes para futuros estudios. En primer lugar, El Agustino ha expuesto la necesidad de analizar cuál es el papel del sector privado en el fomento del uso del espacio público en contextos en los que las gestiones públicas son ineficientes y como consecuencia de los niveles de inseguridad. En segundo lugar, San Isidro ha evidenciado la existencia de un fenómeno que produce la deficiencia de los servicios en zonas periféricas, pero, a su vez, en aquellas en las que el público usuario no es exclusivo. Con ello, permite repensar la delimitación y los efectos de esta en el espectro social. 


\section{Bibliografía}

Alianza Electoral Unidad Nacional. (2007). Plan de Gobierno Municipal: El Agustino 2007- 2011. Recuperado de http://www.infogob.com.pe/General/ObtenerArchivo?fileURL=GbUy ObOtBKNLGxU\%2BGhut4eb12PFwnsiWACDd9ElP@0ZRnv902C

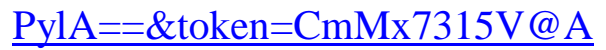

Bensa, Jessica. (2015). «La gobernanza urbana en contextos de descentralización y fragmentación institucional: el caso de Lima. Metropolitana: El Gobierno de las grandes ciudades. Gobernanza y descentralización en las metrópolis de América Latina». Ed. Grin, Eduardo \& Hernández, José. Santiago: CLAD ICHEM, 241- 264.

Col.lectiu Punto 6. (2016). ¿Qué es el urbanismo con perspectiva de género?. Recuperado el 20 de abril del 2017 de http://punt6.org

Diario Gestión. (2017). ¿Cuál es el mejor distrito para vivir en Lima? Recuperado de https://gestion.pe/tendencias/mejor-distrito-vivir-lima$\underline{127118 ? \text { foto }=5}$

El Comercio. (2017). Lima es la $5^{\circ}$ ciudad más peligrosa para mujeres en el mundo. Recuperado de https://elcomercio.pe/lima/sucesos/limaquinta-ciudad-peligrosa-mujeres-mundo-noticia-465995

Espinoza, Álvaro y Fort, Ricardo. (2017). Calidad de la intervención pública sin planificación territorial: ¿Por qué la inversión pública urbana es mal priorizada, desarticulada y fragamentada?. Recuperado de https://saberespoder.lamula.pe/2017/06/07/la-calidad-de-la-inversionpublica-sin-planificacion-territorial/saberespoder/

Fondo Mivivienda. (2015). ¿Qué es el Fondo Mivivienda y cuáles son sus programas?. Recuperado el 12 de abril del 2017 de https://www.mivivienda.com.pe/PORTALWEB/fondo-

MIVIVIENDA/pagina.aspx?idpage $=134$ 
Instituto Nacional de Estadística e Informática (INEI). (2015). Perú: Estimación y proyecciones de Población por Sexo, según Departamento, Provincia y Distrito, 2000 - 2015 ( $\left.\mathrm{N}^{\mathrm{o}} 18\right)$.

Recuperado de https://www.um.es/documents/378246/2964900/Normas+APA+Sexta +Edici\%C3\%B3n.pdf/27f8511d-95b6-4096-8d3e-f8492f61c6dc

Instituto Nacional de Estadística e Informática. (2017). Comportamiento de la tasa de homicidios, 2011 - 2016. (Homicidios En El Perú, Contándolos Uno A Uno).

Jacobs, Jane. (2001). Muerte y Vida de las grandes ciudades. Madrid: Capitán Swing.

López, David. (2014). «Género, Estética y Diseño Urbano ¿Crear espacios para vivir mejor? ». Fòrum de recerca, 2014, No-19: 165-176.

Recuperado de https://doi.org/10.6035/forumrecerca.2014.19.12

Mckenzie, Evans. (1996). Privatopia: homeowner Associations and the Rise of Residential Private Government. Yale University, Connecticut.

Miranda, Carla y Gutiérrez, Juan. (s/f). Políticas urbanas en espacios públicos con enfoque de seguridad ciudadana: el caso del Municipio de Toluca, México. Tesis de maestría. Universidad Autónoma del Estado de México, Ciudad de México.

Municipalidad Distrital de El Agustino. (2013) Plan de Desarrollo Local Concertado hacia el 2021 con Proyección al 2030. Recuperado de http://mdea.gob.pe/files/PDLC_2030_PROYECTO_FINAL.pdf

Muxí, Zaida y otros. (2011). «¿Qué aporta la perspectiva de género al urbanismo? ». Feminismo/s vol. 17, No: 105-129. https://doi.org/10.14198/fem.2011.17.06

Plataforma Urbana. (2016) Jane Jacobs y la humanización de la ciudad. http://www.plataformaurbana.cl/archive/2016/05/04/jane-jacobs-y-lahumanizacion-de-la-ciudad/ 
Político Perú Patria Segura. (2014) Plan de Gobierno Municipal: El Agustino. Recuperado de:

http://aplicaciones013.jne.gob.pe/pecaoe/servicios/planGobierno.asm x/DescargarPdf_Ruta

Sánchez de Madariaga, Inés. (2008) Esquinas Inteligentes: la ciudad y el urbanismo moderno. Madrid. Alianza Editorial.

Sánchez de Madariaga, Inés. (s/f.) Urbanismo con Perspectiva de Género. Sevilla: Instituto Andaluz de la Mujer.

Sennett, Richard. (1996) Flesh and stone: the body and the city in western civilization. London: W. W. Norton \& Company

Tys Magazine. (2016) Jane Jacobs, la maestra de la planificación urbana. Recuperado de http://www.tysmagazine.com/jane-jacobs-la-maestrala-planificacion-urbana/

Unidad Nacional. (2002). Plan de Gobierno Municipal 2002. Elecciones $2003 \quad-\quad 2007.2$ Recuperado de http://www.infogob.com.pe/General/ObtenerArchivo?fileURL=GbUy ObOtBKP9HbocWB5rwSsq0iW48t\%2BFj@\%2BO3ci@6Zkc6\%2B @0ElOxMA==\&token=CmMx7315V@

Unidad Nacional. (2010) Plan de Gobierno: Municipalidad distrital de El Agustino. 